\title{
Prevalence of fosfomycin resistance among ESBL-producing Escherichia coli isolates in the community, Switzerland
}

\author{
Linda Mueller ${ }^{1,2}$ (1) $\cdot$ Cansu Cimen ${ }^{1,3} \cdot$ Laurent Poirel $^{1,2,4} \cdot$ Marie-Christine Descombes $^{5} \cdot$ Patrice Nordmann $^{1,2,4,6}$
}

\begin{abstract}
Our aim was to evaluate the prevalence of fosfomycin-resistant strains among ESBL-producing Escherichia coli isolates recovered from community patients in Switzerland. A total of 1225 ESBL-producing E. coli isolates were collected between 2012 and 2013 from a private and community laboratory. Fosfomycin resistance was assessed by using the novel rapid fosfomycin/E. coli NP test and agar dilution method. Resistant isolates were further investigated for acquired resistance genes fos A1-7 by PCR and sequencing. Pulsed-field gel electrophoresis (PFGE) and multilocus sequence typing (MLST) were performed to evaluate the clonal relationship among fosA3-carrying isolates. Out of the 1225 ESBL-producing E. coli isolates analyzed in this study, 1208 were fosfomycin susceptible while 17 were fosfomycin resistant. No discrepancy was observed between the rapid fosfomycin/ $E$. coli NP test and the agar dilution method taken as the gold standard. Five out of the 17 resistant isolates carried a fos $A$-like gene. No clonal relationship was observed among those isolates. Here, the prevalence of fosfomycin resistance among ESBLproducing E. coli isolates in the community is reported for the first time in Switzerland, being ca. 1.4\%. Among the five isolates carrying a fos A gene, four encoded the FosA3 enzyme, being the most prevalent fosfomycin-resistant determinant. An excellent correlation was observed between minimum inhibitory concentration-based susceptibility categorization and results of the rapid fosfomycin/E. coli NP test, further indicating the excellent sensitivity and specificity of this recently developed rapid test whose results are obtained in less than $2 \mathrm{~h}$.
\end{abstract}

Keywords Rapid test · Enterobacterales

\section{Introduction}

On a global scale, it is estimated that urinary tract infections (UTIs) affect 150 million people per year. Escherichia coli is

Linda Mueller

linda.mueller@unifr.ch

1 Emerging Antibiotic Resistance Unit, Medical and Molecular Microbiology, Faculty of Science and Medicine, University of Fribourg, Chemin du Musée 18, 1700 Fribourg, Switzerland

2 Swiss National Reference Center for Emerging Antibiotic Resistance (NARA), University of Fribourg, Fribourg, Switzerland

3 Infectious Diseases and Clinical Microbiology Clinic, Ardahan Public Hospital, Ardahan, Turkey

4 INSERM European Unit (IAME/LEA, France), University of Fribourg, Fribourg, Switzerland

5 Laboratoire Proxilis, Meyrin, Switzerland

6 University Hospital Center and University of Lausanne, Lausanne, Switzerland the most frequent bacterial species responsible for UTIs. It is estimated that $E$. coli causes $75 \%$ of uncomplicated and $65 \%$ of complicated UTIs [1]. Due to an increased prevalence rate of extended-spectrum $\beta$-lactamases (ESBLs) conferring resistance to broad-spectrum cephalosporins, and due to the occurrence of associated multiple resistance traits in those ESBLproducing isolates, multidrug-resistant isolates are often identified nowadays in those urinary pathogens [2-4].

Fosfomycin is a phosphonic acid-derived antibiotic owning unique structure and mechanism of action, granting for a broad-spectrum activity and without cross-resistance with other antibiotics. For these reasons, fosfomycin is a first-line treatment as oral therapy for uncomplicated UTIs. Fosfomycin shows good activity against $E$. coli isolates, exhibiting a resistance rate estimated to be $<10 \%[5,6]$.

Chromosomally encoded fosfomycin resistance mechanisms in E. coli include reduced drug uptake, due to mutations in the genes encoding GlpT and UhpT transporters, and mutations in the fosfomycin target which is the enzyme catalyzing the first step in peptidoglycan biosynthesis, namely MurA. Reduced drug uptake remains the most frequently 
encountered fosfomycin resistance mechanism not only among clinical isolates but also among in vitro obtained mutants [7, 8].

Transferable resistance mechanisms in E. coli entail the FosA metallo-enzymes, responsible for fosfomycin inactivation by catalyzing the conjugation of glutathione to this antibiotic molecule [8]. Among the seven FosA variants reported so far, four (FosA3, FosA4, FosA5, and FosA6) have been identified as acquired resistance determinants among $E$. coli isolates [9-11]. The FosA3 variant is the most relevant since it has been extensively reported in humans and animals, mainly in Asia but also in Europe and the USA [12-14]. The FosA3 encoding gene is located onto conjugative plasmids coencoding ESBLs of the CTX-M-type [15]. Hence, selective pressure with fosfomycin and/or broad-spectrum cephalosporins may lead to acquisition of those plasmid and consequently to co-resistance to those drugs.

Fosfomycin resistance rates in ESBL-producing E. coli in Europe, Asia, and the USA is estimated to be low, the average being ca. 3.2\% [5]. However, a Spanish study showed an increase in fosfomycin resistance rates among ESBLproducing E. coli from $2.2 \%$ in 2004 to $21.7 \%$ in 2008 , in parallel to a concomitant $50 \%$ increase in fosfomycin consumption [16]. An even higher rate of fosfomycin resistance of $31.1 \%$ in ESBL producers from UTI is now reported from Israel [17]. To estimate the prevalence of fosfomycin resistance in community-acquired ESBL-producing E. coli isolates in Switzerland, we have screened a collection of 1225 isolates recovered in a Swiss laboratory during 2 years. This study was performed in community settings since this is the most important location of $E$. coli spread. The genetic bases of acquired fosfomycin resistance have been deciphered, and the clonal relationship among FosA producers has been evaluated.

\section{Materials and methods}

\section{Isolate collection}

A total of 1225 ESBL-producing E. coli isolates were collected from a Swiss private laboratory between 2012 and 2013. Samples were of different nature, including urine $(\sim 89 \%)$, genital and anal swabs $(\sim 4 \%)$, stool $(\sim 3 \%)$, and blood cultures $(\sim 2 \%)$. The remaining $2 \%$ of the samples were classified as "other" and were of various nature such as expectoration and abscesses. Samples were recovered from women, men, and newborns. Ages ranged from 0 to 103 years.

\section{Fosfomycin resistance determination}

Two techniques were used to assess fosfomycin susceptibility. First, the agar dilution method using cation-adjusted MuellerHinton agar (MHA-CA, reference 64884; Bio-Rad, Marnes-
la-Coquette, France), supplemented with $25 \mu \mathrm{g} / \mathrm{ml}$ of glucose6-phosphate, was used to determine fosfomycin minimum inhibitory concentration (MIC) over a range of dilutions (1$512 \mu \mathrm{g} / \mathrm{ml}$ ) as recommended by Clinical Laboratory Standard Institute (CLSI) guidelines [18]. The breakpoints of the European Committee on Antimicrobial Susceptibility Testing (EUCAST) were used as reference [19]. E. coli isolates with MICs of fosfomycin $\leq 32 \mu \mathrm{g} / \mathrm{ml}$ were categorized as susceptible while those with MICs $>32 \mu \mathrm{g} / \mathrm{ml}$ were categorized as resistant. Uncertain MICs as well as MICs close to the breakpoint were repeated three times. Second, the rapid fosfomycin/E. coli NP test was performed as described in Nordmann et al. [20]. Results were read after $1 \mathrm{~h}$ and $30 \mathrm{~min}$ of incubation at $35 \pm 2{ }^{\circ} \mathrm{C}$. Agar dilution method and rapid fosfomycin/E. coli NP results were further compared.

\section{FosA role in fosfomycin resistance}

The contribution of FosA resistance proteins in fosfomycin resistance was assessed by using the disk diffusion method as described in Nakamura et al. [9]. Briefly, 0.5 McFarland standard of fosfomycin-resistant $E$. coli isolates were inoculated onto Mueller-Hinter agar plates supplemented with $25 \mu \mathrm{g} / \mathrm{ml}$ of glucose-6-phosphate. Two Kirby-Bauer disks containing $200 \mu \mathrm{g}$ of fosfomycin, with and without $0.5 \mathrm{mg}$ of the FosA-inhibitor sodium phosphonoformate (PPF) (Sigma-Aldrich), were added on the plates, which were incubated at $35 \pm 2{ }^{\circ} \mathrm{C} \mathrm{O} / \mathrm{N}$. Actually, PPF inhibits selectively the FosA proteins that allows differentiation between plasmidmediated FosA and chromosome-encoded resistance mechanisms to fosfomycin. An increase in the diameter of the growth inhibition zone by $\geq 5 \mathrm{~mm}$ in the presence of PPF is interpreted as a FosA-related resistance.

\section{PCR amplification and sequencing}

Isolates of DNA were extracted with the QIAamp DNA Mini Kit and the QIAcube workstation (Qiagen, Courtaboeuf, France), according to the manufacturer's instructions. PCR amplification followed by sequencing (Microsynth, Balgach, Switzerland) was performed to detect the plasmid-mediated fos A genes (fosAl to fosA6) as previously described [20]. The presence of $f o s A 7$ gene was investigated with the following primers: fosA-7_Fw (5'-TGTGGCGACCTTTG GGTCTG-3'), fosA-7 Rv (5'-AGTTCCAGCTTGTG GCCATC-3'). PCR conditions were the same as those used for fosA1-6 gene amplification and described previously.

FosA3-carrying isolates were further screened for CTX-Mtype ESBL presence. Primers CTX-M-A1 (5'-SCS ATG TGC AGY ACC AGT AA-3') and CTX-M-A2 (5'-GCC GCC GAC GCT AAT ACA TC-3') were used to amplify bla $a_{\mathrm{CTX}-\mathrm{M}-1 \text {-like }}$ genes (including $b l a_{\mathrm{CTX}-\mathrm{M}-3}$ and $b l a_{\mathrm{CTX}-\mathrm{M}-15}$ ), and primers CTX-M-9-F (5'-GGT GAC AAA GAG AGT GCA AC-3') 
and CTX-M-9-R (5'-GCT GGG TAA AAT AGG TCA CC-3') were used to amplify the $b l a_{\mathrm{CTX}-\mathrm{M}-9}-\mathrm{like}$ genes. To detect the $b l a_{\text {CTX-M-8 }}$ and $b l a_{\text {CTX-M-2 }}$ genes, primers CTX-M-8-F (5'ATG AGA CAT CGC GTT AAG CG-3') and CTX-M-8-R (5'-CGT CGT ACC ATA ATC ACC GC-3'), or primers CTX-M-2-F (5'-TGA TGA CTC AGA GCA TTC GC-3') and CTX-M-2-R (5'-AAG TAG GTC ACC AGA ACC AG$3^{\prime}$ ), were used, respectively. PCR conditions were $95^{\circ} \mathrm{C}$ for 5 min, followed by 30 cycles of $95^{\circ} \mathrm{C}$ for $30 \mathrm{~s}, 52^{\circ} \mathrm{C}$ for $30 \mathrm{~s}$, and $72{ }^{\circ} \mathrm{C}$ for $30 \mathrm{~s}$, with a final extension of $72{ }^{\circ} \mathrm{C}$ for $5 \mathrm{~min}$. Sequences were analyzed with CloneManager Professional (Sci-Ed Software, Denver, CO, USA).

\section{Clonal relationship}

Clonal relationship of the bacterial isolates was evaluated by pulsed-field gel electrophoresis (PFGE). Total DNAs from five $E$. coli isolates carrying a plasmid-encoded fos $A 3$ gene were digested using XbaI enzyme (New England BioLabs, Ipswich, MA, USA). PFGE was then assessed using the CHEF-DR III System (Bio-Rad) to separate the digestiongenerated fragments. Finally, multilocus sequence typing (MLST) was assessed on all E. coli isolates carrying a FosA-like encoding gene and sequence types (STs) assigned according to the EnteroBase database (http://mlst.warwick.ac. $\mathrm{uk} / \mathrm{mlst} / \mathrm{dbs} /$ Ecoli/).

\section{Results}

A total of 1225 ESBL-producing $E$. coli isolates were investigated for fosfomycin resistance. Seventeen isolates turned to be positive according to the results of the rapid fosfomycin/ E. coli NP test. Precise determination of their MIC values showed that, for those strains, fosfomycin MICs ranged from 64 to $>512 \mu \mathrm{g} / \mathrm{ml}$, further confirming the categorization of those isolates as being truly fosfomycin resistant (Table 1). Among them, four isolates possessed the fos 33 gene and a single isolate possessed the fos 4 . Two out of four isolates carrying a fos $A 3$ gene co-harbored a $b l a_{\mathrm{CTX} \text {-M-3 }}$ gene, one isolate possessed a $b l a_{\mathrm{CTX}-\mathrm{M}-15}$ gene, and another single isolate possessed the $b l a_{\text {CTX-M-9 }}$ gene (Table 1). Twelve isolates remained negative for all the fos $A 1-7$ genes by PCR. Four out of the five isolates possessing a fos $A$ gene showed accordingly an increased diameter of the growth inhibition in the presence of the FosA-inhibitor PPF. Noticeably, two isolates showing increased diameters around the fosfomycin disk with PPF remained negative for the fosAl-7 genes (Table 1). Other mechanisms of resistance to fosfomycin were not further investigated.

The 1208 fosfomycin-susceptible isolates exhibited MICs ranging between 1 and $32 \mu \mathrm{g} / \mathrm{ml}$ and accordingly remained negative for the rapid fosfomycin/E. coli NP test. Overall, it is interesting to highlight that results of susceptibility testing and of the rapid fosfomycin/E. coli NP test showed $100 \%$ correlation, for both susceptible and resistant isolates. Among them, 15 isolates exhibited MIC values close to the breakpoint value $(n=3$, MICs of fosfomycin at $64 \mu \mathrm{g} / \mathrm{ml} ; n=4$, MICs at $128 \mu \mathrm{g} / \mathrm{ml} ; n=8$, MICs at $32 \mu \mathrm{g} / \mathrm{ml})$, but nevertheless gave concordant results with the rapid test.

PFGE and MLST analyses showed no clonal relationship among the fosfomycin-resistant isolates producing a FosA enzyme. More precisely, the four isolates carrying the fos $A 3$ gene were of sequence types ST117, ST359 (ST101 complex), ST69, and ST10, while that carrying the fosA4 gene was of ST12 (Table 1).

\section{Discussion}

This is the first study evaluating the prevalence of fosfomycin resistance among ESBL-producing E. coli in Switzerland. Our results showed a low prevalence in the community, being ca. $1.4 \%$. This prevalence rate is lower than that reported previously (a total of 11 distinct studies from 2004 to 2009) from the USA, Asia, and Europe where the fosfomycin resistance rate had been estimated to be $3.2 \%$ [5]. Even though studies published after 2010 reported a slight increase in fosfomycin resistance, the estimated rate is still considered to be below $10 \%$ [6]. This lower fosfomycin resistance rate observed among community-acquired pathogens in Switzerland may mirror the overall low resistance levels observed for many pathogens in Switzerland (www.anresis.ch). Additionally, differences in fosfomycin use might also reflect differences in fosfomycin resistance. A former Swiss study evaluating antimicrobial resistance among $E$. coli isolates recovered from E. coli in a tertiary care hospital between 2012 and 2015 reports a fosfomycin resistance rate of $0.9 \%$ [21]. However, the prevalence fosfomycin rate among ESBL vs non-ESBL E. coli producers had not been evaluated.

Considering the general low prevalence of fosfomycin resistance observed in this study and in other studies performed from 2004 to 2015 [5, 6], we may speculate that there is an overall low risk of rapid expansion of fosfomycin resistance among ESBL-producing E. coli. Nevertheless, the analysis reported in the current study was performed on samples collected between 2012 and 2013, and might not reflect the contemporary fosfomycin resistance rate. Further studies are therefore needed to confirm a low fosfomycin resistance among ESBL-producing E. coli in Switzerland in 2019.

Our data highlights that $29 \%$ of the fosfomycin-resistant ESBL-producing E. coli isolates possess a fos $A$ gene. These isolates were clonally unrelated, exhibiting different clonal profiles and sequence types, suggesting that acquired plasmid-encoded FosA-related resistance occurred through independent genetic events. 
Table 1 Features of the fosfomycin-resistant ESBL-producing E. coli

\begin{tabular}{|c|c|c|c|c|c|c|c|c|}
\hline $\begin{array}{l}\text { Fosfomycin } \\
\text { phenotype }\end{array}$ & $\begin{array}{l}\mathrm{MIC} \\
(\mu \mathrm{g} / \mathrm{l})\end{array}$ & $\begin{array}{l}\text { Rapid fosfomycin/ } \\
\text { E. coli NP test }\end{array}$ & $\begin{array}{l}\text { PPF } \\
\text { potentiation } \\
\text { test }\end{array}$ & $\begin{array}{l}\text { FosA } \\
\text { determinant }\end{array}$ & $\begin{array}{l}\text { ESBL } \\
\text { determinant }\end{array}$ & $\begin{array}{l}\text { Number of } \\
\text { isolates }\end{array}$ & Sequence type & $\begin{array}{l}\text { Year of } \\
\text { isolation }\end{array}$ \\
\hline $\mathrm{R}$ & $>512$ & + & - & fos $A 3$ & CTX-M-3 & $n=1$ & ST10 & 2012 \\
\hline $\mathrm{R}$ & $>512$ & + & + & fos $A 3$ & CTX-M-3 & $n=1$ & ST117 & 2013 \\
\hline $\mathrm{R}$ & $>512$ & + & + & fos $A 3$ & СТХ-М-9 & $n=1$ & $\begin{array}{c}\text { ST359 (ST101 } \\
\text { complex) }\end{array}$ & 2013 \\
\hline $\mathrm{R}$ & $>512$ & + & + & fos $A 3$ & CTX-M-15 & $n=1$ & ST69 & 2013 \\
\hline $\mathrm{R}$ & $>512$ & + & + & fosA4 & $\mathrm{Na}$ & $n=1$ & ST12 & 2013 \\
\hline $\mathrm{R}$ & $>512$ & + & $n=1+n=1-$ & - & $\mathrm{Na}$ & $n=2$ & NA & 2013 \\
\hline $\mathrm{R}$ & 256 & + & - & - & $\mathrm{Na}$ & $n=3$ & NA & 2013 \\
\hline $\mathrm{R}$ & 128 & + & $n=1+n=3-$ & - & $\mathrm{Na}$ & $n=4$ & NA & 2013 \\
\hline $\mathrm{R}$ & 64 & + & - & - & $\mathrm{Na}$ & $n=3$ & NA & 2012,2013 \\
\hline
\end{tabular}

MLST was assessed in FosA-producing E. coli isolates only

$N A$ not applicable, $N a$ not assessed

The FosA3 enzyme was first identified in Japan, from an E. coli isolate collected between 2002 and 2007 [12]. In 2010, this enzyme was observed in an E. coli isolate in the USA [13], then in France [15] and in Switzerland (this study) in 2012, highlighting its worldwide dissemination. Intriguingly, similar to one isolate from 2013 carrying the fos $A 3$ gene detected in our study, another FosA3-producing ST69 E. coli had been recovered in France in 2014, suggesting a clonal relationship [15].

As expected, the majority ( $80 \%$ ) of FosA-producing E. coli analyzed in our study carried a fos 33 gene, confirming that this is the most prevalent plasmid-mediated fosfomycin resistance gene variant among $E$. coli, including among ESBL producers. Isolates carrying a FosA3-encoding gene also carried produced CTX-M-type ESBLs, being of either CTX-M3-, CTX-M-15-, or CTX-M-9-types, further highlighting that associations between $f o s A 3$ and $b l a_{\mathrm{CTX}-\mathrm{M}}$ genes are frequent, as widely reported [12, 14, 22].

Noteworthily, two E. coli ESBL-producing isolates exhibited an increased inhibition zone diameter on the agar plate in the presence of PPF (positive result), but remained negative for $f o s A 1-7$ amplification, suggesting the possible production of still unknown FosA-related or FosA-unrelated resistance mechanism(s).

Surprisingly, for a single E. coli isolate carrying a fosA3 gene, the PPF test remained negative, suggesting that the phenotypic resistance observed might be resulting from both a plasmid-encoded and a chromosomally encoded resistance mechanism(s). The other possibility is that this PPFmediated inhibition might lack the sensitivity for detecting FosA-related resistance to fosfomycin.

It has been suggested that mutations in several chromosomal genes involved in fosfomycin resistance in $E$. coli clinical isolates might have an elevated biological cost, therefore explaining the overall low prevalence of fosfomycin resistance in E. coli [14]. By contrast to chromosomal mutations, production of a FosA-like enzyme has been estimated to generate a lower biological cost and therefore may be prone to be easily disseminated [23].

Finally, this study represents the first extended survey evaluating the performance of the rapid fosfomycin/E. coli NP test for rapid screening of fosfomycin resistance in a large collection of clinical isolates. Thanks to its excellent performance, this test whose results are given in less than $2 \mathrm{~h}$ and is low cost might be widely implemented to accurately monitor the evolution of fosfomycin resistance rates worldwide and is adapted to rapid determination of fosfomycin susceptibility for an adapted antibiotic stewardship of septicemia.

Funding information This work was funded by the Swiss National Science Foundation (project FNS-407240 177382).

\section{References}

1. Flores-Mireles AL, Walker JN, Caparon M, Hultgren SJ (2015) Urinary tract infections: epidemiology, mechanisms of infection and treatment options. Nat Rev Microbiol 13(5):269-284

2. Pitout JDD, Laupland KB (2008) Extended-spectrum $\beta$-lactamaseproducing Enterobacteriaceae: an emerging public-health concern. Lancet Infect Dis 8(3):159-166

3. Ayad A, Drissi M, de Curraize C, Dupont C, Hartmann A, Solanas $S$ et al (2016) Occurence of ArmA and RmtB aminoglycoside resistance 16S rRNA methylases in extended-spectrum B-lactamases producing Escherichia coli in Algerian hospitals. Front Microbiol 7:1409

4. Lupo A, Saras E, Madec JY, Haenni M (2018) Emergence of bla $a_{\mathrm{CTX}-\mathrm{M}-55}$ associated with $f o s A, r m t B$ and $m c r$ gene variants in Escherichia coli from various animal species in France. J Antimicrob Chemother 73(4):867-872

5. Falagas ME, Kastoris AC, Kapaskelis AM, Karageorgopoulos DE (2010) Fosfomycin for the treatment of multidrug-resistant, including extended-spectrum $\beta$-lactamase producing, Enterobacteriaceae infections: a systematic review. Lancet Infect Dis 10:43-50 
6. Falagas ME, Vouloumanou EK, Samonis G, Vardakas KZ (2016) Fosfomycin. Clin Microbiol Rev 29(2):321-347

7. Castaneda-Garcia A, Blazquez J, Rodriguez-Rojas A (2013) Molecular mechanisms and clinical impact of acquired and intrinsic fosfomycin resistance. Antibiotics. 2(2):217-236

8. Silver LL (2017) Fosfomycin: mechanism and resistance. Cold Spring Harb Perspect Med 7(2):a025262

9. Nakamura G, Wachino J, Sato N, Kimura K, Yamada K, Jin W et al (2014) Practical agar-based disk potentiation test for detection of fosfomycin-nonsusceptible Escherichia coli clinical isolates producing glutathione S-transferases. J Clin Microbiol 52(9):31753179

10. Ma Y, Xu X, Guo Q, Wang P, Wang W, Wang M (2015) Characterization of fos $A 5$, a new plasmid-mediated fosfomycin resistance gene in Escherichia coli. Lett Appl Microbiol 60(3):259264

11. Guo Q, Tomich AD, McElheny CL, Cooper VS, Stoesser N, Wang $\mathrm{M}$ et al (2016) Glutathione-S-transferase FosA6 of Klebsiella pneumoniae origin conferring fosfomycin resistance in ESBLproducing Escherichia coli. J Antimicrob Chemother 71(9):24602465

12. Wachino J, Yamane K, Suzuki S, Kimura K, Arakawa Y (2010) Prevalence of fosfomycin resistance among CTX-M-producing Escherichia coli clinical isolates in Japan and identification of novel plasmid-mediated fosfomycin-modifying enzymes. Antimicrob Agents Chemother 54(7):3061-3064

13. Alrowais H, McElheny CL, Spychala CN, Sastry S, Guo Q, Butt AA et al (2015) Fosfomycin resistance in Escherichia coli, Pennsylvania, USA. Emerg Infect Dis 21(11):2045-2047

14. Yao H, Wu D, Lei L, Shen Z, Wang Y, Liao K (2016) The detection of fosfomycin resistance genes in Enterobacteriaceae from pets and their owners. Vet Microbiol 193:67-71

15. Benzerara Y, Gallah S, Hommeril B, Genel N, Decre D, Rottman M et al (2017) Emergence of plasmid-mediated fosfomycin-resistance genes among Escherichia coli isolates, France. Emerg Infect Dis 23(9):1564-1567

16. Oteo J, Orden B, Bautista V, Cuevas O, Arroyo M, Martinez-Ruiz R et al (2009) CTX-M-15-producing urinary Escherichia coli O25bST131-phylogroup B2 has acquired resistance to fosfomycin. J Antimicrob Chemother 64(4):712-717

17. Peretz A, Naamneh B, Tkhawkho L, Nitzan O (2019) High rates of fosfomycin resistance in Gram-negative urinary isolates from Israel. Microb Drug Resist In press 0(0):1-5

18. Institute CaLS (2018) Performance standards for antimicrobial susceptibility testing; 28th informational supplement. CLSI document M100-S28. Clinical and Laboratory Standards Institute, Wayne, PA

19. European Committee on Antimicrobial Susceptibility Testing (2019) Breakpoint tables for interpretation of MICs and zone diameters. Version 9.0. http://www.eucast.org/fileadmin/src/ media/PDFs/EUCAST files/Breakpoint tables/v 9.0 Breakpoint_Tables.pdf Accessed 10 jan 2019

20. Nordmann P, Poirel L, Mueller L (2019) Rapid detection of Fosfomycin resistance in Escherichia coli. J Clin Microbiol 57(1):e01531-e01518

21. Erb S, Frei R, Tschudin Sutter S, Egli A, Dangel M, Bonkat G et al (2018) Basic patient characteristics predict antimicrobial resistance in E. coli from urinary tract specimens: a retrospective cohort analysis of 5246 urine samples. Swiss Med Wkly 148:w14660

22. Lee SY, Park YJ, Yu JK, Jung S, Kim Y, Jeong SH et al (2012) Prevalence of acquired fosfomycin resistance among extendedspectrum ß-lactamase-producing Escherichia coli and Klebsiella pneumoniae clinical isolates in Korea and IS26-composite transposon surrounding fosA3. J Antimicrob Chemother 67(12):28432847

23. Cattoir V, Guérin F (2018) How is fosfomycin resistance developed in Escherichia coli? Future Microbiol 13(16):1693-1696 